\title{
An Efficient Self-Service Pre-Payment Courier System on Mobile NFC Technology
}

\author{
Bin Wang ${ }^{1}$, Jun $\mathrm{Guo}^{1}$ and Dong $\mathrm{Su}^{2}$ \\ ${ }^{1}$ College of Electronic Engineering \\ Chongqing University of Posts and Telecommunications \\ 226th Institute of Electronics Technology Group Corporation \\ Chongqing, China \\ wangbin1@cqupt.edu.cn
}

\begin{abstract}
The business and payment can be achieved not only using real money but also virtual one. With the rapid development of e-commerce, the virtual one will be more convenient for transaction. Therefore, an SSPP (Self-Service Pre-Payment) courier system is presented, which characterizes an innovative, convenient and efficient NFC (Near Field Communication) pre-payment system based on card simulation mode. The pre-payment process concerning virtual currency has been supported through a simple NFC device, which operates in RF (Radio Frequency) band. It ensures the micro-payment services of mobile to POS can be achieved. In this paper, an efficient scheme is described to check its feasibility, and some experimental results have also been given to verify that this system is candidate for the future express industry.
\end{abstract}

Keywords : pre-payment; NFC; SSPP; card simulation mode

\section{Introduction}

People's daily lives are inseparable from deal, such as, travel, buying tickets, group purchase etc. The combination of NFC with smart devices resulted in widening the range of NFC, which includes data exchange, service discovery, connection, e-payment, and ticketing. It is expected to replace credit cards in electronic payment, especially. According to Gartner, a market research company, the number of NFC-based payment services is expected to increase by 11.3 times from $\$ 316$ million in 2010 to $\$ 3.572$ billion in 2015 [1], and Juniper predicted that the global NFC payment market size would be increased to $\$ 180$ billion in 2017 [2].

Considering such a huge market, the traditional express industry is suffering from a rapid decline. At present, the domestic express industry cannot adapt to the rapid development of electronic business in some areas, and a number of $\mathrm{B} 2 \mathrm{C}$ electronic business enterprises have begun to establish their own logistics systems.

Express has been widely used by people. Every day, the post office and courier business outlets accumulate a large number of parcels. Therefore, the SSPP courier system has another serious problem for the rapidly growing express market. Thus, it plays a very important role in accelerating the formation of an intelligent, efficient express system. NFC mobile payment technology reflects its importance, especially in the traditional courier industry.

Currently, NFC technology has been applied to many aspects of the logistics industry, including cargo container tracking and management, distribution center management etc. However, it still does not achieve the fast delivery and payment of the express perfectly.

The purpose of this paper is to design this system using the NFC technology and take a specific test for the read or write mode based on operating system of Android. 


\section{Background Review}

\subsection{Overview of NFC-enabled Mobile Payment}

NFC is a wireless communication technology that enables data transmission over a distance of $10 \mathrm{~cm}$ through a combination of RFID and contactless smart cards technologies [3]. It was the brainchild of Philips and Sony companies, which founded the NFC Forum. NFC protocol uses the $13.56 \mathrm{MHz}$ carrier frequency that enables the devices to transfer data at $424 \mathrm{kbps}$ [4]. An NFC device can operate in three modes: read/write, peer-to-peer and card emulation mode. The read/write mode enables an NFC device to read and exchange data passively on NFC transponders like NFC tags, however, the card emulation mode enables the NFC devices to function as a smart card in which the data could be read by an external reader. Last but not least, the peer to-peer mode allows transmit and receive the data actively or simultaneously between two NFC devices. NFC technology can be applied in a variety of industries including transportation, finance, manufacturing, automobile, advertising, healthcare, etc.

In China, with the rapid development of economy and science, the payment system is being extended from wired to wireless, and NFC mobile payment technique enters a new period of development. According to Enfodesk, a data analysis organization, the number of internet users has reached 500 million by the end of 2012 in China. At that time, the number of mobile phone users reached 921 million, and mobile internet users reached 320 million. What is the more, the number of online shopping has reached 242 million, an annual growth rate of $66.5 \%$.

Up to now, many NFC trials have been conducted all over the world, especially in payment domain. All the trials get a fact that mobile phone tends to become faster, safer, more convenient and more fashionable with the development of NFC technology. It allows person to integrate their daily-use transportation cards, credit cards etc. into their mobile phones. In addition to integrating these cards into their mobile devices, NFC technology brings the innovation opportunities to mobile payment field.

\subsection{NFC Payment on the Cloud}

As we know SE (Secure Element) is a requirement for the secure storage and operating of NFC enabled applications especially in card emulation mode [5]. The SE is the combination of software, hardware, and protocols, which embedded in a mobile handset that enables data secure storage. Currently, many SE alternatives are being considered, and the current alternatives are Embedded Hardware based SE, SMC (Secure Memory Card) based SE, UICC (Universal Integrated Circuit Card) based SE, and Software based SE.

The data is mostly created by some M-payment (Mobile payment) related activity. But it is often in dangerous, when the mobile phone is been stolen or lost, it means that a bit potential risk on maintaining local data will be happened. In this study, we have been motivated for enhancing the capability of SE by moving its location to the cloud, and improve the processing capabilities.

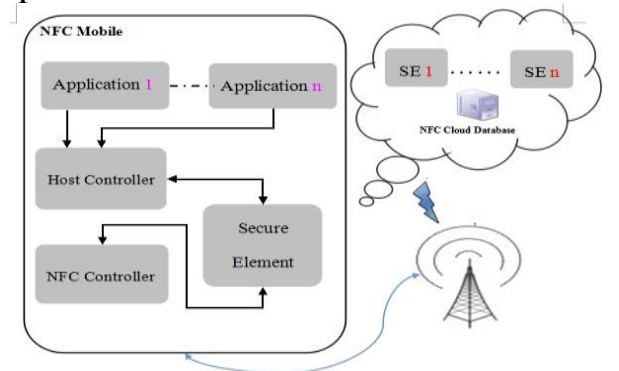

Figure 1. NFC Payment Architecture and SE on the Cloud 
Our model is depicted in Figure 1. In this model, SE is created and maintained on the cloud, which can be accessed through the security protocol. When any service on the mobile phone is invoked, the respective service will contact with the local host controller on the mobile, which will enable the communication with the SE on the cloud afterwards.

\section{Design and Application}

\subsection{Design of Hardware Circuits}

Some PN series chips for NXP have become the mainstream of NFC enabled mobile phones, as shown in Figure 2(a). We show the main NFC chip named PN544 embedded in the phone and its peripheral circuits. The PN544 chip is full featured NFC controller designed for integration in mobile phones. It is optimized for low power consumption with fully host controllable power states and low footprint for mobile phone applications.

The 27.12MHz clock applied to the PN544 is the time reference for the RF front-end when PN544 is in reader mode or ISO/IEC 18092 initiator mode. Therefore stability of the clock frequency is an important factor for reliable operation [6]. It is recommended to adopt the circuit shown in Figure 2(b). And we use 5V's USB power to supply the chip, but choose a $3.3 \mathrm{~V}$ low voltage chip in order to meet the power requirements of the circuit.

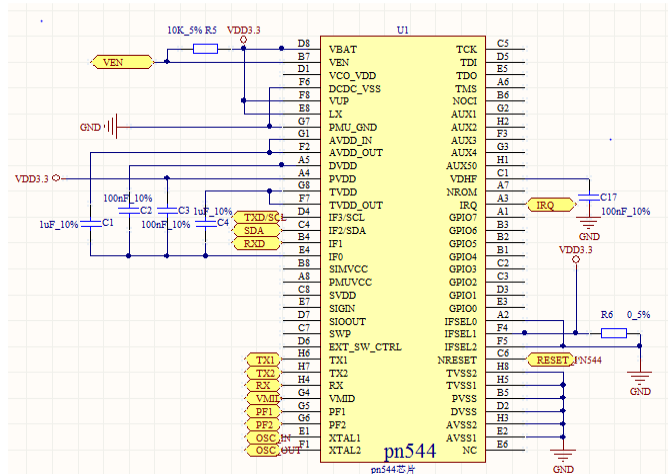

(a) NFC Controller for PN544

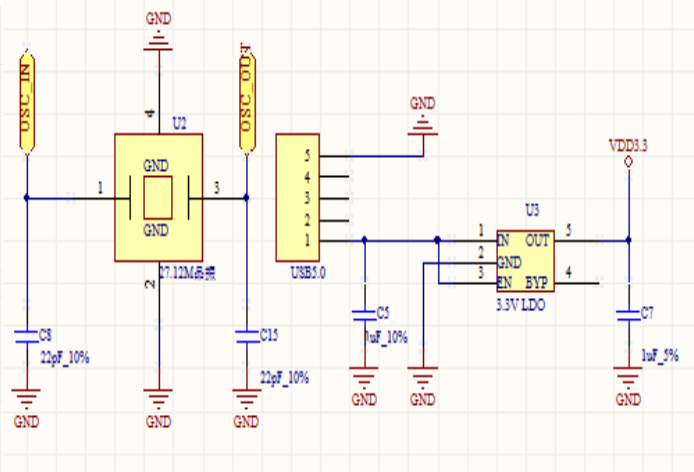

(b) 27.12MHz Oscillator and 3.3V LDO

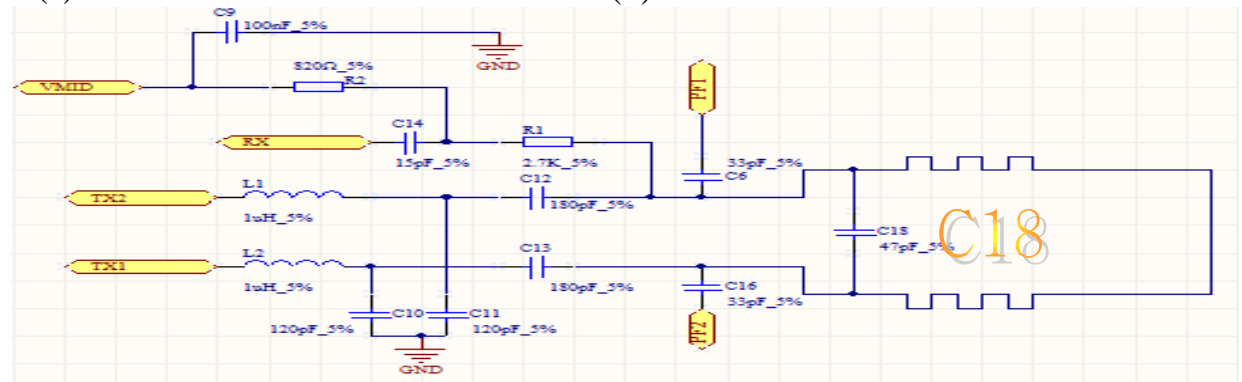

(c) Antenna and Its Matching Circuit

Figure 2. The Chip and Peripheral Matching Circuits

In Figure 2(c), we can see the antenna for sending and receiving RF fields and the corresponding matching circuit, and we should change the capacitance of $\mathrm{C} 18$ to get a better performance of antenna. It is known that the development of contactless smart cards and NFC devices have been growing rapidly for several years. In particular, mobile phones equipped with NFC technology open up the door with a variety of new NFC applications.

This step is important to maximize the power of the emitted RF field. Therefore, it is vital to maximize the range of the transmission module and the quality of the transmitted signal, while keeping the power consumption of the transmitter low. 


\subsection{A Creative Solution for NFC Antenna}

There is no doubt that the antenna acts an important role in NFC communication, which determines the communication effect and communication distance due to the basic parameters. What is more, the performance of NFC system is dependent on NFC antenna, so a new method of antenna processing is proposed for NFC mobile payment in this paper.

As we know, there are too many approaches to process an NFC antenna layout on a selected substrate. The earliest and most inconvenient method of antenna manufacture has been subtractive etching on a metal plated laminate (e.g., FR-4). It has become a low-tech process requiring inexpensive、 ready-made materials and equipment. However, when larger production runs, the etching will bring a large amount of metal and chemical waste, incurring increased costs.

Contrary to the traditional etching technique, the inkjet printing technology is a direct-write technology by which the designed pattern is transferred directly to the substrate [7]. This technology has been become a major project in scientific research domain, especially drop-on-demand (DOD) inkjet printing systems [8]. With the developing of this new technology, the production costs of electronic devices are dramatically reduced, as material can be deposited on demand.

It is estimated that there are more 1500 worldwide research units are working on various aspects of flexible electronic device [9]. The manufacture of thin conductive tracking by method of inkjet printing has been recently considered widely [10-11].

In the actual fabrication process, several parameters will be involved, such as substrate, size of ink nanoparticles, time, temperature of ink sinter and surface tension of the particle etc. Among others [12], which provide an extensive range of freedom for circuit designs, and particularly for the antenna. Future terminals, such as smart phones, are envisaged to be completely flexible in the near future [13-15]. The antenna has a considerable size in the system, therefore, its flexibility is crucial if a flexible terminal is desired.

The usage of inkjet technology for NFC implementation on flexible terminals still finds another motivation for the antennas involved. The $\mathrm{Q}$ factor required for antenna is directly related to the read range, and it is achieved by the inkjet technology. For example, in a typical RFID system, for a distance of $36 \mathrm{~cm}$, the $\mathrm{Q}$ factor is required to close to 40 [16, 17]. As NFC allows for shorter distances motivated for a whole sort of reasons, for example, as a result of security and privacy of users, the restriction on $\mathrm{Q}$ factor is relaxed to get the same voltage for energizing the RFID tag integrated the circuit. The $\mathrm{Q}$ factor of spiral inductor is then given as:

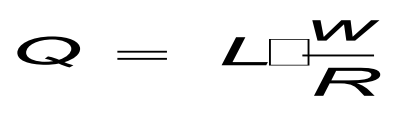

The thickness of conductive track deposited by inkjet printing technology can be adjusted micron by micron as the number of layers. If the skin-effect at $13.56 \mathrm{MHz}$ is approximately $46 \mu \mathrm{m}$ for the silver conductive ink, the input resistance varies inversely with the thickness according to the following expression:

$$
R=\frac{1}{\delta} \square \frac{L}{S}=\frac{1}{\delta} \square \frac{L}{t \leftarrow W}
$$

Where $\mathrm{R}$ is the electrical resistance, $\delta$ is the ink's conductivity, $\mathrm{S}$ is the track cross section, $\mathrm{L}$ is the longitude, $\mathrm{w}$ is the width of the track, and $\mathrm{t}$ is the thickness. 
So, an exploration of the potentials and limitations of inkjet printing technology for NFC flexible terminals should be concentrated on this context since it provides a low cost, easy manufacturing process on flexible materials, no complex resistance involved.

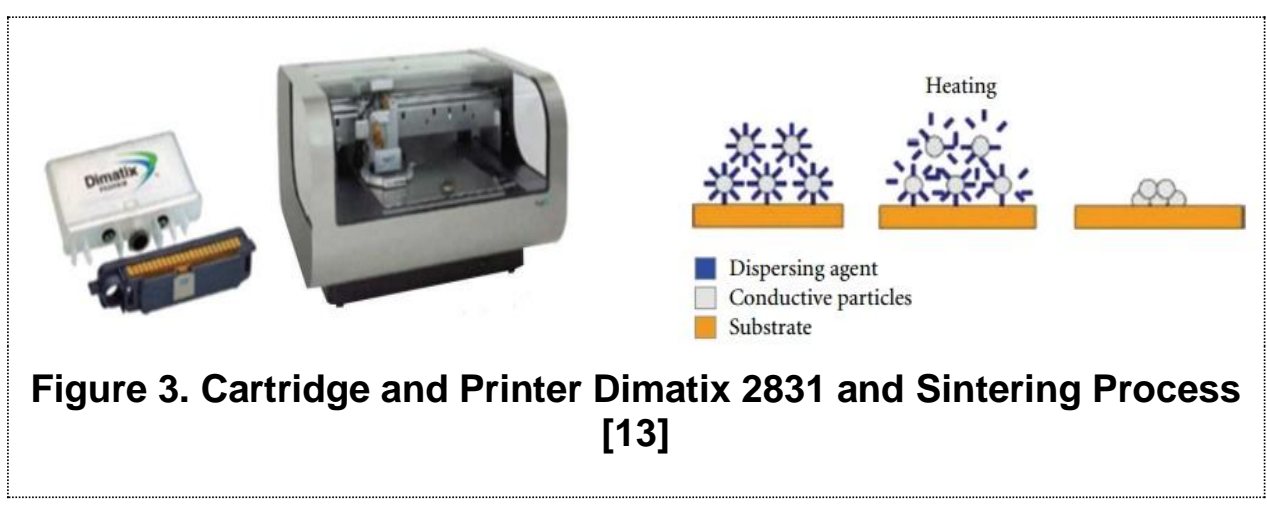

In this paper, the printer which has been chosen is the Dimatix Materials Printer DMP-2831 (Figure 3). The platen temperature can be varied from $28{ }^{\circ} \mathrm{C}$ to $60^{\circ} \mathrm{C}$. In the fabricated prototypes, $60{ }^{\circ} \mathrm{C}$ is chosen as it prevents the ink from spreading out. At last, a 6 layers NFC antenna made by DMP-2831 is shown in Figure 4.

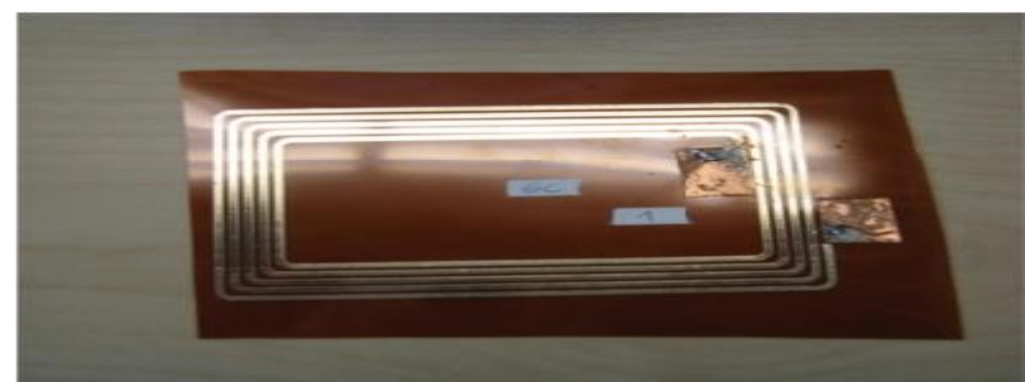

Figure 4. Printed Prototype (6 Layers)

The huge potential of using inkjet printing technology to process NFC antennas has been considered. A general scheme for the design, simulation, process, and measurement has been proposed. The resistance can be controlled by the technology of varying the track thickness, which prevents from extra resistor implementations. Take advantage of the aforementioned solution, the $\mathrm{Q}$ value ranged from 4 to 8 can be obtained while keeping the same inductance value.

Therefore, inkjet printing technology is a promising technique for the manufacture of NFC coil antennas. Moreover, due to its low cost of process and substrate flexibility, it is the most appropriate choice for the fabrication of NFC antennas embedded intelligent NFC terminals.

\subsection{Fabrication and Application of This System}

According to the hardware circuit we have given, we drew the PCB layout of NFC module, as shown in Figure 5. 


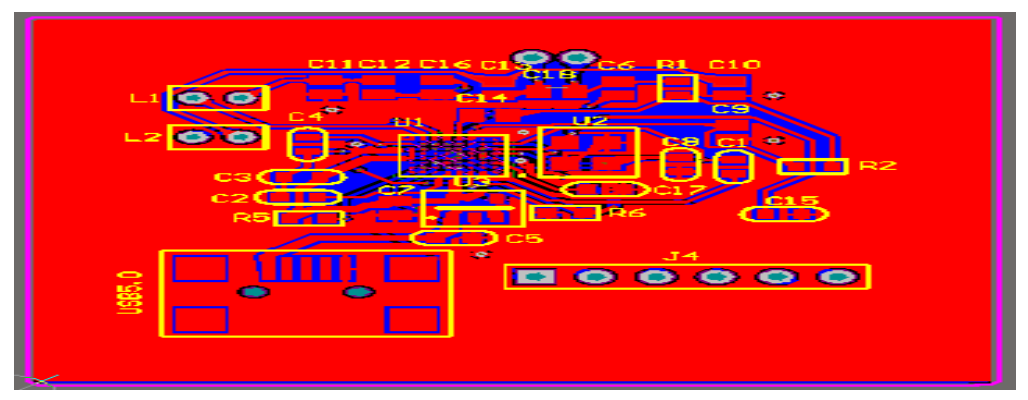

Figure 5. The PCB Layout of NFC Module

The NFC module will be connected to antenna for the next test. We have compiled the "NFC-USB" drive, and the following interface is also given in Fig.6. Selecting the appropriate serial port number, the acquiescent serial port parameters and supported card, we click on "Open NFC device" button to open the corresponding serial ports, and the NFC test board will be initialized.
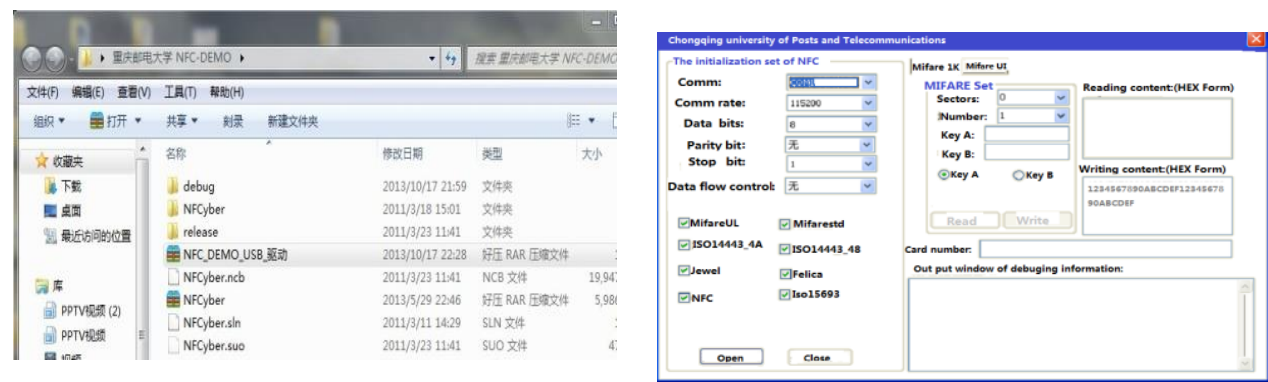

Figure 6. The File and Interface of NFC Tool

We can choose Mifare1K card (it is also known as NFC tag) and enter the password of corresponding RF card in the edit box of password A and B, choosing to use key A for authentication or key B for certification.

If you want to read or write the data in a certain sector, simply select the appropriate sector number and block number, and click on "Read Card" or "Write Card". Then make the card approach the antenna. If the write data is correct, the button will be automatically enabled. Otherwise, it will keep the prohibited state.

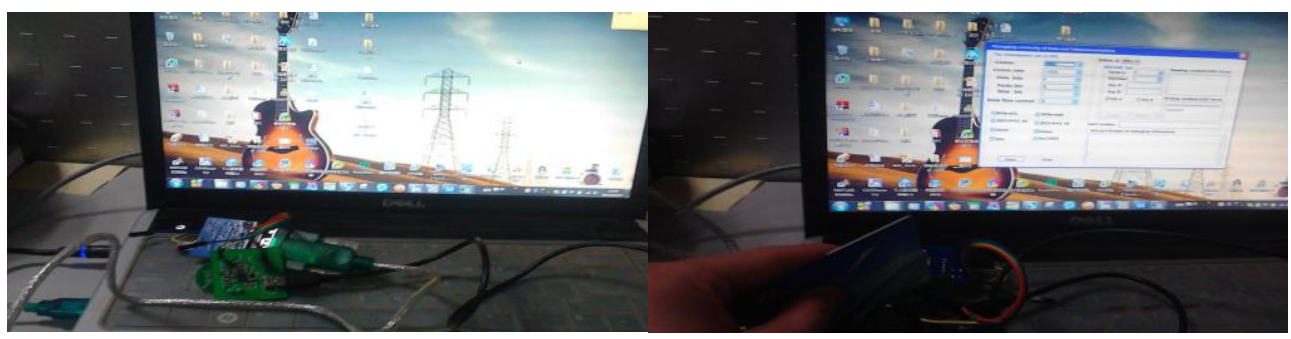

Figure 7. The Information of NFC Tag is being Written

For the SSPP courier system, we can write the receiver's own message number to a NFC tag. So when you receive and send a courier, just gently close to a reader, your information will display on the computer of server through the reader. In order to prepare for the complex experiments later, we take advantage of this hardware and software device to write the URLs of "http://www.nfc-forum.org" to a tag, as shown in Figure 7. We can see a tag is being close to a NFC phone in Figure 8. And we can use the card 
reader mode of NFC for gathering information. At the same time, the message is being detected, so it proves that it is successful to write the tag.

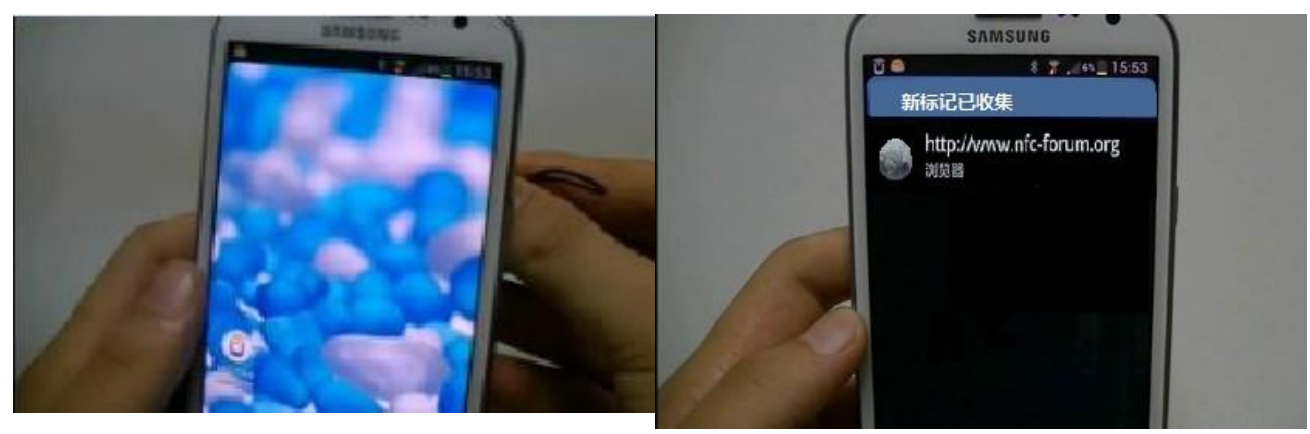

Figure 8. The Collection of Tag Information

What the next work to be done is achieving its NFC function in Android development

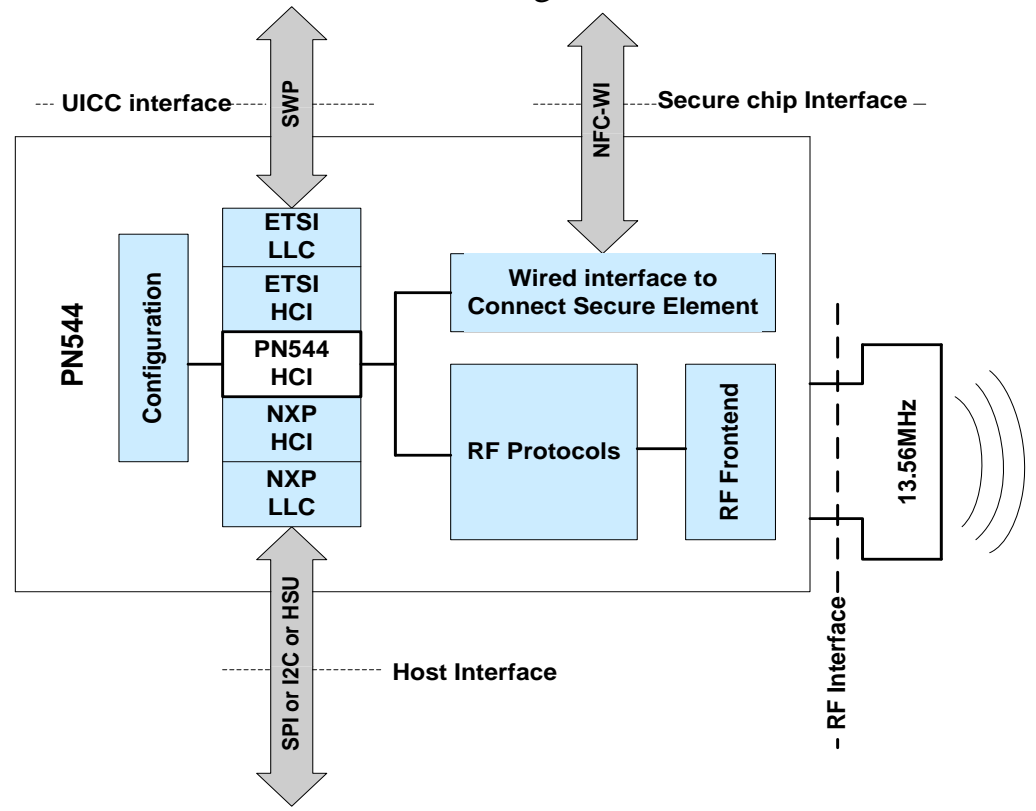

Figure 9. The Working Diagram of PN544

board, and we can use a PN544 chip with its peripheral circuits and equip with Android 4.0 development board. The main communication mode of host platform and PN544 chip is sent ETSI HCI message from the host to the chip, and it is also transmitted through the LLC. In Fig.9, the PN544 chip makes use of four interfaces of LLC on the physical layer including host interface (I2C, SPI and HSU) and ESTISWP interface. So the NFC module can equip to an Android 4.0 development board through the $\mathrm{I} 2 \mathrm{C}$ interface for the latter experiments and test.

\section{Analysis and Discussion}

\subsection{Characteristics between Old Courier Industry}

For the traditional courier industry, it is inevitable to complete sign, checking information, payment and other operations. For example, when you are ready to mail an expedited parcel to Beijing, inevitably, you have to follow the traditional delivery 
processes, fill out the address, zip code, telephone and all other details. It is known that China is a populous country, according to the data of China Electronic Commerce Research Center, there are about 25 million parcels every day, along with a lot of paper and manpower waste. But the current situation will be able to get change if we have a NFC enabled phone.

\subsection{The Specific Process}

Considering these situation, we should try to achieve the global automation and intelligence. In fact, logistics are closely linked with our lives. There are many domestic express enterprises, but they can't satisfy our needs because of lower efficiency. The corresponding industry norms and standards are also imperfect. For such an emerging NFC application, it is inextricably linked with the network. So we can build a professional logistics website for NFC mobile payment technology to serve everyone, and each person can apply for an exclusive ID. The most important thing is to bind with the phone and make your own registration information into the phone via NFC tags with its reader/writer mode. What is more, the information can be displayed through the reader to the PC. So each transaction will be recorded, and you can monitor and track parcels every moment.

For the operation of pre-payment, you can pay through your own online banking, or use a NFC enabled phone to make payments via POS machines where are equipped with the express outlets.

When you walk into any one of the outlets, the moment you contact with a NFC reader, the reader which was connected to the PC terminal will show you the information registered on the web site. At the same time, your information is incorporated into the two -dimensional bar code posted with the parcel for sender. Similarly, just tell the staff the ID of receiver, the receiver information will also be loaded into the two-dimensional bar code. At this point, your account will generate a deal which shows the parcel sender and receiver, at the same time, the corresponding account will receive some real information about the parcel synchronously and wait for reception. All information will be uploaded to the server via the "cloud" count, and you can log in your account, check the pack on any courier outlets.

When a user changes his work address, he can log in his own network account in a prompt manner or go to the lobby to change his account information, and the system will be updated in time.

Logistics Center will scan the two-dimensional code by hand-held devices, and do the correct allocation, so the corresponding parcel will be sent to all affiliated outlets. Obviously, each delivery outlets will receive a lot of packages every day, so how to distinguish these parcels quickly and easily will be a major problem. Today, however, express outlets' staffs just re-scan the two-dimensional bar code which was attached to the parcel, upload the data to PC, then arrange and number these parcels. When a receiver reaches outlets, just gently close to the NFC reader connected to the PC terminal, it will display some basic information about the account, so we can achieve the identity recognition more quickly. At the same time, it will show the parcel number to enter the ID of sender, so as to quickly find the corresponding courier.

\subsection{Application Prospect}

With the arrival of big data, the future network development will play a major role. The establishment of this system promotes the formation of express industry standards better, and also bring the huge business value. Reference [18] predicts that 863 million units, or $53 \%$ of new handsets will be NFC-enabled until 2015. Whether online payment or mobile payment used NFC enabled phone will increase the number of third-party transactions. With the cooperation of major electricity supplier, customers want to buy a product from electricity supplier website, just clicking a chaining to jump to the professional express 
websites we have built, through price comparison, logistics speed, customer evaluation, etc., then select a cost-effective logistics companies. On the one hand, the system can promote the resource integration and the formation of healthy competition for express industry. On the other hand, we will gain huge consumer groups for the most valuable resource. In China, the e-commerce which is an emerging industry will continue to replace some of the traditional business model, so it will eventually become the main force of economic development in the future. Apparently, this innovative system we have proposed will be an extremely important e-commerce application.

\section{Conclusions}

In this paper, a new SSPP courier system is proposed on NFC technology which can improve the efficiency of the express industry in order to meet people's needs. Although the application of mobile payment shows some weaknesses in terms of security, it provides higher usability due to the intuitive and seamless acquisition of all kind of monitoring data. Summarizing the specific test work of the designed system, we also think the system could be used in present life. It is the next step to come out the application of the designed system.

\section{Acknowledgment}

This work was supported by the Natural Science Foundation of CQ CSTC under Grant cstcjjA40022, the Science and Technology Research Project of Chongqing Municipal Educational Commission of China under Grant KJ120505, and 2013 University Innovation Team Construction Plan Funding Project of Chongqing.

\section{References}

[1] Gartner, "Market Insight: The Outlook on Mobile Payment," Market Analysis and Statistics, May (2010).

[2] Juniper Research, "NFC Mobile Payments \& Retail Marketing-Business Models \& Forecasts 2012-2017," (2012) May.

[3] G. Madlmayr, "A Mobile Trusted Computing Architecture for A Near Field Communication Ecosystem", in Proceedings of the 10th international conference on information integration Web-based applications \& services, (2008) September, pp. 563-566.

[4] N. Chidembo, "Exploring Consumer Adoption of NFC-enabled Mobile Payments in South Africa", Published master thesis, University of Pretoria, (2009), South Africa.

[5] V. Coskun and B. Ozdenizci, "A Survey on Near Field Communication (NFC) Technology", Journal of Wireless Personal Communications, published online, DOI: 10.1007/s11277-012-0935-5.

[6] The objective data sheet of PN544 for NXP company.

[7] A. Rida, R. Vyas, L. Yang, C. Kruesi, and M. Tentzeris, "Low Cost Inkjet-printing Paper-based Modules for RFID Sensing and Wireless Applications," in Proceedings of the 38th European Microwave Conference, (2008) October, pp. 1715-1718, Amsterdam, Netherlands, .

[8] T. H. J. van Osch, J. Perelaer A. W. M. de Laat, and U.S. Schubert, "Inkjet Printing of Narrow Conductive Tracks on Untreated Polymeric Substrates," Advanced Materials, vol. 20, no. 2, (2008), pp. 343-345.

[9] J. Hu, "Overview of Flexible Electronics from ITRI's Viewpoint," in Proceedings of the 28th IEEE VLSI Test Symposium(VTS'10), Santa, Cruz, Calif, USA, (2010) April, p. 84.

[10] M. L. Allen, K. Jaakkola, K. Nummila and H.Seppa, "Applicability of Metallic Nanoparticle Inks in RFID Applications," IEEE Transactions on Components and Packaging Technologies, vol. 32, no. 2, (2009), pp. 325-332.

[11] S. H. Yoon, J. H. Lee and P. C. Lee, "Sintering and Consolidation of Silver Nanoparticles Printed on Polyimide Substrate Films," Macromolecular Research, vol. 17, no. 8, (2009), pp. 568-574.

[12] R. D. Mancosu, J. A. Q. Quintero, and R. E. S. Azevedo, "Sintering in Different Temperatures of Traces of Silver Printed in Flexible Surfaces," in Proceedings of the 11th International Conference on Thermal, Mechanical and Multi-Physics Simulation, and Experiments in Microelectronics and Micro systems, (2010), pp. 1-5, April, Bordeaux, France.

[13] "Samsung and Nokia Are Moving towards with Flexible Screens Smartphone," Available at: http://www.mobiussite.com/samsung--nokia-moving-flexible/1830.html. 
[14] "Samsung Flexible Display Phones\&Tablets in 2012," Available at: http://www.slashgear.com /samsung-flexible-display-phones-tabletsin-2012-28191673.

[15] Y. Min, "Mobile Terminal Using Flexible Display and Method of Controlling The Mobile Terminal," U.S. Patent US 2010/0117975 A1, (2010).

[16] A. L. Scholtz and R. Weigel, "Antenna Design for Future Multi-standard and Multi-frequency RFID Systems", (2009).

[17] Youbok Lee, "Antenna circuit design for RFID applications," Microchip, (2003), September, pp. 1-50.

[18] B. Frost, and J. Sullivan, "When Will Be the Real Start for NFC", Plenum Publishers, (2011) November, Australia.

\section{Authors}

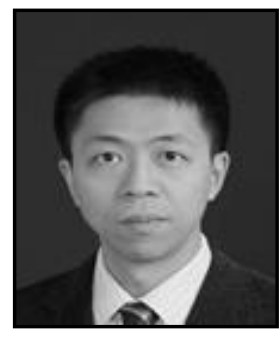

Bin Wang, he was born in 1982. He received the B.S. and M.S. degrees from Sichuan University in 2004 and 2007 respectively, and the Ph.D. degree from the Institute of Electronics, Chinese Academy of Sciences in 2010. He is an associate professor and the master's supervisor at Chongqing University of Posts and Telecommunications. His current research interests include RF communication and high-power millimeter-wave technologies.

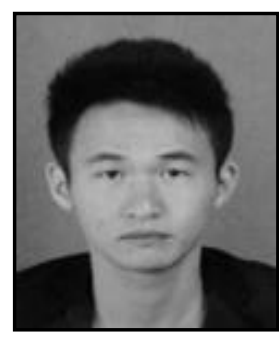

Jun Guo, he was born in 1989. He received his B.S. degree from Chu Zhou College in 2008. Currently, he is a Master degree candidate in Chongqing University of Posts and Telecommunications, China. His recent research interests include RF communication and wireless transmission technologies.

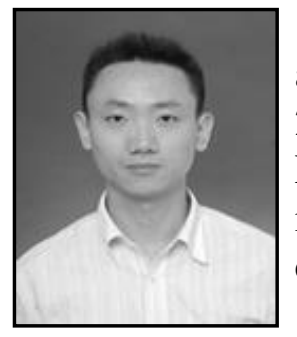

Dong Su, he was born in 1983. He received the B.S., M.S. and Ph.D. degrees from Sichuan University in 2004, 2007 and 2012 respectively. He is an Electronic Engineer at the China Electronics technology Group corporation 26th research institute. His current research interests include RF communication and radar technologies. 